\title{
Ground-penetrating radar for tree trunk investigation
}

\author{
Jana Ježová and Sébastien Lambot \\ Earth and Life Institute \\ Université catholique de Louvain \\ Louvain-la-Neuve, Belgium \\ Email: jana.jezova@uclouvain.be, \\ sebastien.lambot@uclouvain.be
}

\author{
Alessandro Fedeli and Andrea Randazzo \\ Department of Electrical, Electronic, \\ Telecommunications Engineering, and Naval Architecture \\ University of Genoa, Genoa, Italy \\ Email: alessandro.fedeli@edu.unige.it, \\ andrea.randazzo@unige.it
}

\begin{abstract}
Tree trunk inspection is a very important task to predict possible collapses of trees and thus prevent harming people and damages to infrastructure. To perform such investigation non-invasively, ground-penetrating radar (GPR) appears to be a very promising tool. The objective of this paper is to test a frequency-domain radar system with a new home-made transverse electromagnetic (TEM) horn antenna to investigate the internal structure of a laboratory model of a tree trunk. In order to test the horn antenna, a calibration was performed to determine its transfer functions following the intrinsic antenna model of Lambot et al.. Subsequently, a radar profile was acquired over a sand box to test its imaging capabilities. Finally, a circumferential acquisition around the laboratory model with different antenna-medium distances was performed. Results were compared to a classical time-domain radar acquisition. The new acquired data showed very good GPR profiles, so the horn antenna proved to be a suitable tool for our measurements. In the end, influence of a polar representation of an irregular tree trunk was discussed and the structure from motion (SfM) technique was proposed to acquire a correct shape of an irregular cross section.
\end{abstract}

\section{INTRODUCTION}

Trees are a very important part of everyday life. Therefore, it is necessary to study their condition carefully to predict their possible collapses which can endanger people and urban infrastructures. Tree trunk inspection is a complicated discipline due to a generally complex internal structure, which is anisotropic and heterogeneous. Furthermore, the electromagnetic properties of living wood depend on its moisture, density and on the frequency of the applied field [1]. Several techniques are currently under development for the electromagnetic characterization of wooden samples. For instance, in [2] a microwave imaging system was applied to retrieve the spatial distribution of the dielectric properties of wooden structures. Tree trunk investigation is also complicated due to roughness of the bark and also often very irregular shape of the tree trunk cross section.

In order to non-invasively analyse the internal structure of a tree trunk, Ježová et al. [3] used ground-penetrating radar (GPR) for tree trunk testing with a commercial time-domain ground-coupled radar system as a powerful non-destructive device. GPR was used in many different fields [4], such as civil and transport engineering [5], [6], archaeology [7], soil moisture mapping [8], [9], and progressively also wooden samples and tree trunk observation [10].

GPR operates with various kinds of antennas with significant restrictions to fulfil conditions for achieving a high value of range resolution, such as large fractional bandwidth, low time side lobes, and, in the case of separate transmitting and receiving antennas, low cross coupling levels. A transverse electromagnetic (TEM) horn antenna has been widely used as a very powerful ultra-wideband structure [11]. It is characterised by large bandwidth, good directivity, no dispersion, and easy construction [12]. A conventional air-filled TEM horn antenna consists of two waveguides (metal plates, wires, etc.) connected to a coaxial cable [13]. The tapering of the plates gradually changes in order to increase the impedance of the antenna from $50 \Omega$ at the antenna throat, to match with the characteristic impedance of a standard coaxial cable, up to $377 \Omega$ at the aperture, to match with the air.

In this study, a TEM horn antenna for a frequency-domain system was designed, made and tested in order to compare its efficiency for a tree trunk investigation with a commercial time-domain radar system. In that respect, the TEM horn antenna was calibrated in order to determine its transfer functions following the antenna model of Lambot et al. [14]. Then, a radar profile was acquired over a sandbox to test the performances of the antenna. Finally, a circumferential profile was acquired around a laboratory tree trunk model, and the ideal distance of the antenna from the model surface was analysed. Then, the obtained GPR image was compared with time-domain radar measurements.

Finally, the polar representation of an irregular tree trunk cross section was studied and discussed. The structure from motion (SfM) technique was suggested as a promising tool to gain a real shape of a tree trunk. The functionality of this method was analysed and the protocol of its usage was proposed.

\section{Materials AND Methods}

\section{A. Antenna design}

In our previous research [1] and [3], we used a commercial time-domain radar system GSSI SIR-20 (Geophysical Survey System, Inc., Salem, Massachusetts, USA) with a $900 \mathrm{MHz}$ 
centre frequency ground-coupled antenna (bowtie) for a real tree trunk and a laboratory tree trunk model investigation. Although we obtained satisfactory results, data acquisition was not easy to carry out because of the size, shape and weight of the antenna. In order to perform such a measurement more easily, the usage of a lighter antenna with a more convenient shape was proposed.

Because of a suitable shape and a very easy construction, TEM horn antenna appeared to be an excellent trade-off between the antenna performance and the weight. The shape of the antenna was built according to the design proposed by Mallahzadeh and Karshenas [15]. Equation (1) describes the exponential separation between two parallel plates in the $x$ direction, where $d_{0}$ and $d_{L}$ are the separation distances between the plates and $L$ is the length of the horn, which is determined as $L=0.4 \cdot \lambda$, where $\lambda$ is the wavelength in air of the lowest operating frequency (see Fig. 1a). The lowest operating frequency was set to $1.5 \mathrm{GHz}$ in order to keep the size of the antenna as small as possible, but to maintain the frequency range suitable for enough tree trunk penetration.

$$
d\left(x_{i}\right)=d_{0} \cdot \exp \left(x_{i} \cdot \frac{1}{L} \cdot \ln \left(\frac{d_{L}}{d_{0}}\right)\right)
$$

Equation (2) defines the width of the metal plates in $x$ direction, where $Z\left(x_{i}\right)$ is characteristic impedance and $\eta$ is the intrinsic impedance of the free space $(120 \pi \Omega)$,

$$
w\left(x_{i}\right)=\frac{d\left(x_{i}\right)}{Z\left(x_{i}\right)} \cdot \eta
$$

The impedance variation within the antenna can be linear, exponential, Chebyshev, Hecken, etc. [11]. We chose linear increasing of the characteristic impedance (from 50 to $120 \pi \Omega$ ) in our design to evenly favour radiation of all frequencies in the operating range of the antenna. The list of all characteristic impedances and all dimensions is shown in Table I. Fig. 1b shows the $x y$ view of the curved plates and Fig. 1c shows the $x y$ view of the not curved plates of the antenna.

TABLE I: Dimensions of the TEM horn antenna.

\begin{tabular}{ccccc}
\hline Section & $x_{i}[\mathrm{~mm}]$ & $Z\left(x_{i}\right)[\Omega]$ & $w\left(x_{i}\right)[\mathrm{mm}]$ & $d\left(x_{i}\right)[\mathrm{mm}]$ \\
\hline \hline 1 & 0 & 50.0 & 19.6 & 2.6 \\
\hline 2 & 31.8 & 159.0 & 20.8 & 8.8 \\
\hline 3 & 63.7 & 268.0 & 41.7 & 29.6 \\
\hline 4 & 95.5 & 376.8 & 10.0 & 10.0 \\
\hline
\end{tabular}

FEKO - EM Simulation Software was used to simulate the designed antenna characteristics. Fig. 2 shows the frequency dependent complex ratio S11 (simulated with FEKO) of the antenna for a simple case (only two metal plates) and a complex case (two metal plates with the connector).

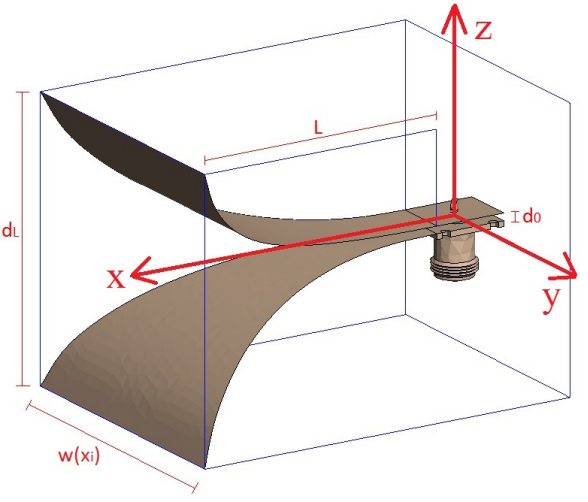

(a)

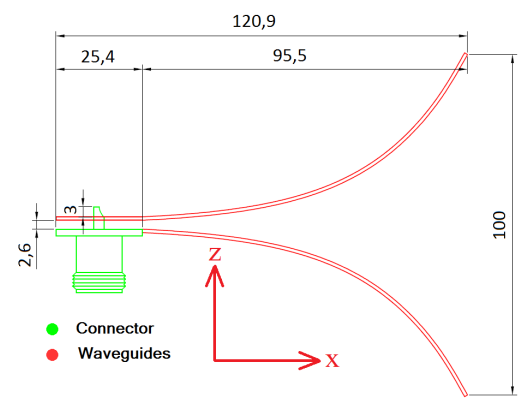

(b)

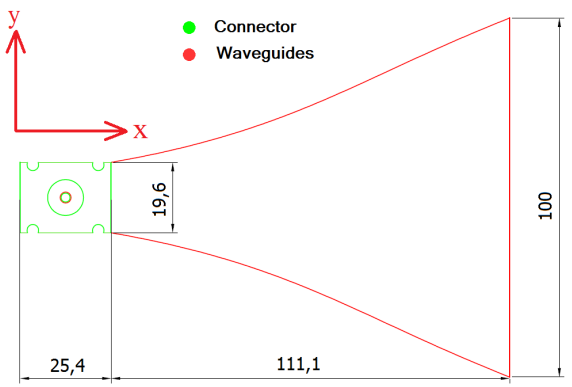

(c)

Fig. 1: TEM horn configuration. (a) 3D view, (b) our design $x z$ view (curved), (c) our design $x y$ view (not curved).

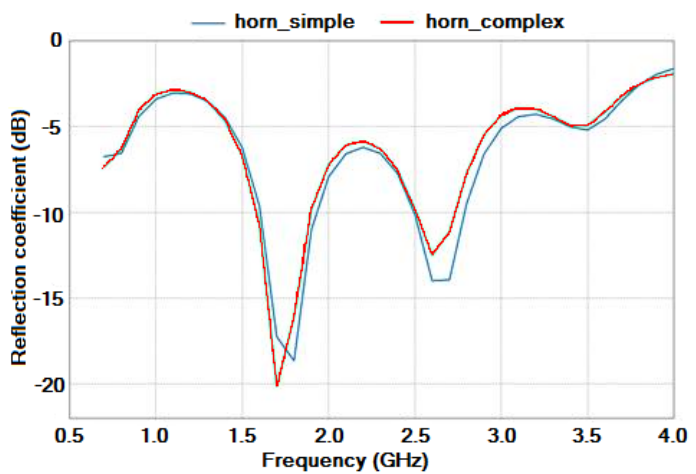

Fig. 2: Amplitude of the free space reflection coefficient S11 of the antenna simulated using FEKO. 


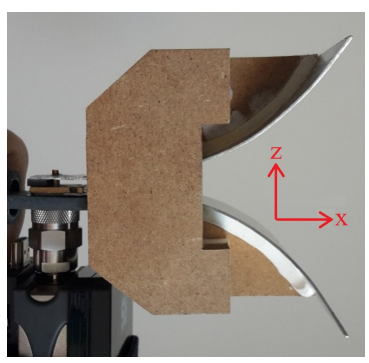

(a)

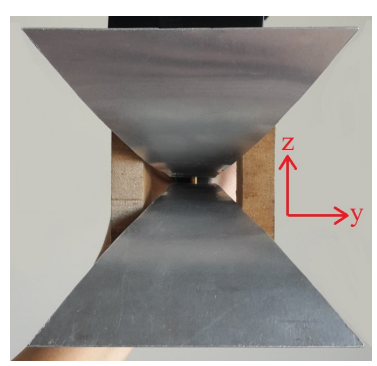

(b)
Fig. 3: Home-made antenna: (a) $x z$ view, (b) $y z$ view.

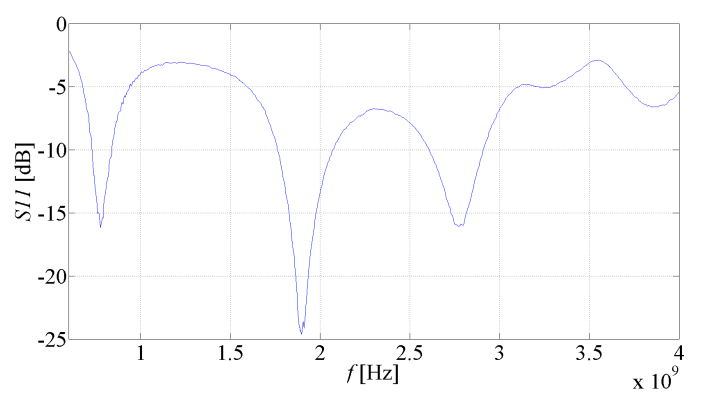

Fig. 4: Measured S11 of the antenna.

The antenna was made from two aluminium plates with a thickness of $1 \mathrm{~mm}$, which were connected to a $50 \Omega \mathrm{HU}$ BER+SUHNER female connector. It is supported by a wooden construction for keeping the correct shape and protecting the antenna (see Fig. 3).

\section{B. Antenna calibration}

For the homemade antenna control, a vector network analyser (VNA, ZVRE, Rhode \& Schwarz, Munich, Germany) was used. The antenna was connected to the VNA with two joined $50 \Omega$ impedance coaxial cables of a length of $2.5 \mathrm{~m}$. The VNA was calibrated using an Open-Short-Match reference calibration kit at the connection between the antenna and the coaxial cable. The operating frequency was set to $0.6-4 \mathrm{GHz}$ (in order to see the antenna performance also below the lowest operating frequency $1.5 \mathrm{GHz}$ ) with a $2 \mathrm{MHz}$ frequency step.

The antenna calibration was performed by means of several measurements at different distances from a copper sheet $(3 \times 3 \mathrm{~m})$. The distance of the antenna varied from 0 to $25 \mathrm{~cm}$ with 100 steps (from 17 to $25 \mathrm{~cm}$ for the far-field) for a frequency range from 0.6 to $4 \mathrm{GHz}$. Fig. 4 shows the measured frequency dependent complex ratio S11, which is comparable with the simulated one in Fig.2.

\section{Antenna testing over a sandbox}

From the calibration results it was clear that the antenna is able to radiate and receive signal satisfactorily. Nevertheless, it was necessary to test the efficiency of the antenna for buried objects detection. In that respect, we buried five plastic pipes (empty, filled by water or filled by wet sand) in a sandbox $(3 \times 3 \mathrm{~m})$. Fig. 5 shows positions of all buried pipes in the

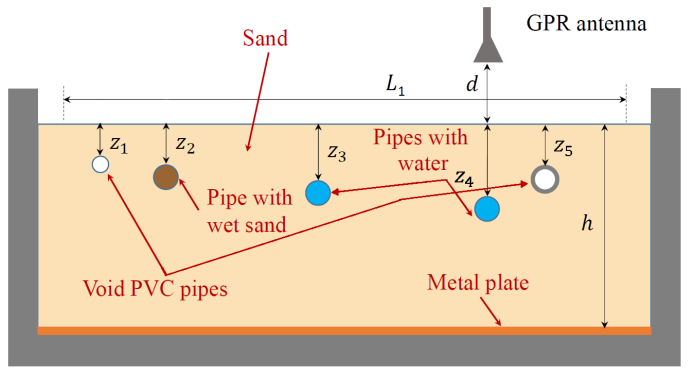

Fig. 5: Location of the pipes in the sandbox.

sandbox and Table II displays a list of their properties, where $D$ is the diameter of the pipe, and $z_{i}$ is the distance between the top of the pipe and the surface. The sandbox is filled by dry sand and its depth $h$ is about $1 \mathrm{~m}$. At the bottom of the box, a $3 \times 3 \mathrm{~m}$ metal plate is situated as bottom boundary condition to prevent reflections from underlying materials. A radar profile of length $L_{1}=2.1 \mathrm{~m}$ was acquired at the distance $d=10 \mathrm{~cm}$ between the antenna aperture and the surface.

TABLE II: Properties of the pipes buried in the sandbox.

\begin{tabular}{cccc}
\hline Cylinder & Material & $D[\mathrm{~mm}]$ & $z_{i}[\mathrm{~mm}]$ \\
\hline \hline 1 & Air & 40 & 125 \\
\hline 2 & Wet sand & 80 & 155 \\
\hline 3 & Water & 80 & 160 \\
\hline 4 & Water & 80 & 175 \\
\hline 5 & Air & 90 & 130 \\
\hline
\end{tabular}

The obtained radar image is shown in Fig. 6. The reflection hyperbolas from all buried pipes are clearly visible. For the airfilled pipes ( 1 and 5$)$ there is just one hyperbola corresponding to the top of the pipe, but the hyperbolas corresponding to the bottom of the pipes are not visible. It can be caused by the small size of the pipe 1 and by the too thick wall of the pipe $5(12 \mathrm{~mm})$. On the contrary, there are two hyperbolas for the pipe 2. The delay of the reflection of the bottom was caused by wet sand with volumetric moisture $\theta_{w}=24 \%$. We can see only one hyperbola corresponding to pipes 3 and 4 , which are filled by water and thus most of the waves were reflected at the top of the pipe. The metal plate at the bottom of the sandbox (10 ns) is well visible. In the GPR image, we can also observe significant side-effects from the edges of the box (under the PEC reflection and on the sides of the GPR image). During the data processing, the antenna effects were filtered out according to Lambot et al. [14] to obtain a clear GPR image.

\section{Laboratory tree trunk model measurement}

The measurement around the tree trunk model was done in order to compare it with our previous data acquisition 


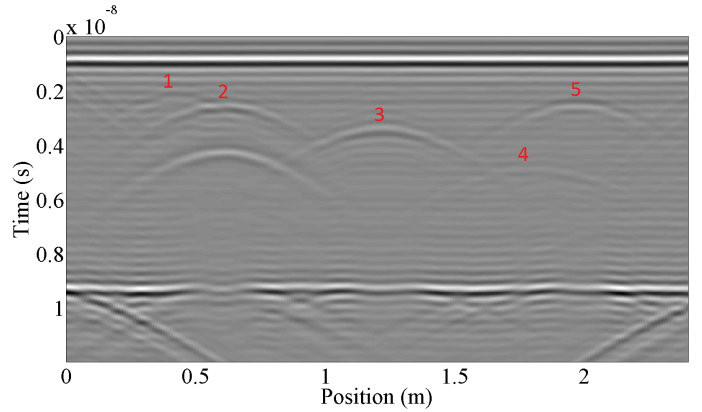

Fig. 6: B-scan over the sandbox with buried pipes.

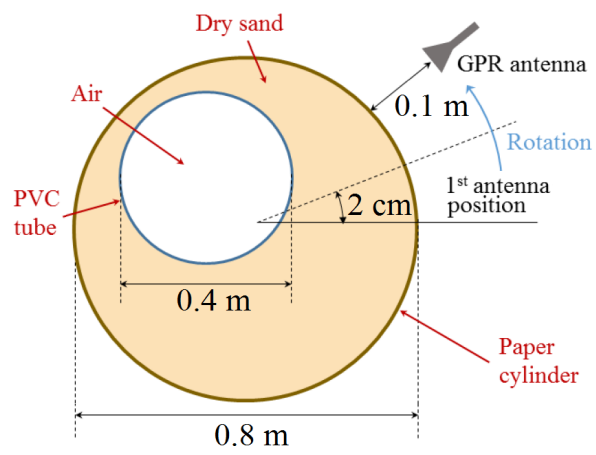

Fig. 7: Sketch of the tree trunk model (horizontal cross section).

with the use of the GSSI time-domain radar system. For that measurement, a $900 \mathrm{MHz}$ centre frequency ground-coupled antenna was used and the data were obtained by a continuous movement around the model. The antenna was always in contact with the surface of the model (but never with a good contact because the antenna is planar and the model is circular).

The new measurement was performed with our new TEM horn antenna and with the VNA (ZVRE, Rhode \& Schwarz). The operating frequency range for this measurement was 0.8 $4 \mathrm{GHz}$ with a step of $2 \mathrm{MHz}$. The sketch of the model is displayed in Fig. 7. The space between the paper and the PVC tubes was filled by dry sand. We obtained the data separately every $2 \mathrm{~cm}$ around the model. The distance between the antenna aperture and the model surface was $d=10 \mathrm{~cm}$, as it appeared to be the most convenient distance for the GPR image quality.

In Fig. 8 the GPR images from both experiments are shown for their comparison. In the first case, the antenna filter was not applied, because the commercial radar system was used. Despite a different data acquisition, some similarities can be observed. In both cases, two reflections from the internal pipe (front and back side) are visible after the surface reflection. Then, opposite side of the model is slightly visible in time $10 \mathrm{~ns}$ in the first case and in time 8-9 ns in the second case. The total internal reflection can be observed in time 14-15 ns in the first case and in time 12-13 ns in the second case.

The GPR image obtained with the TEM horn antenna

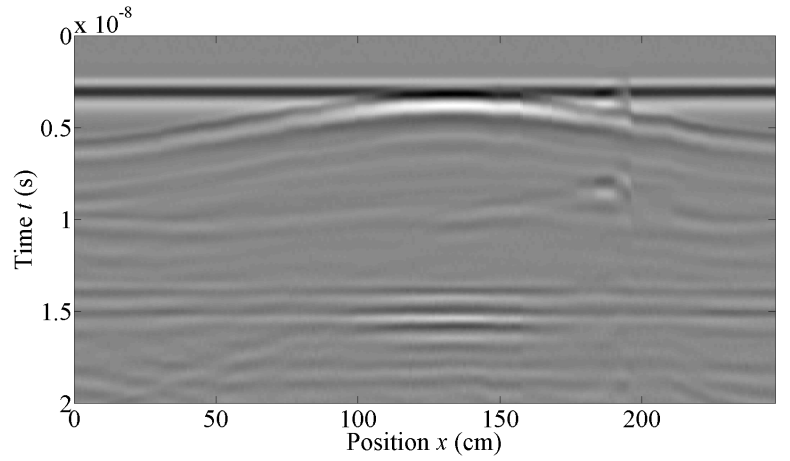

(a)

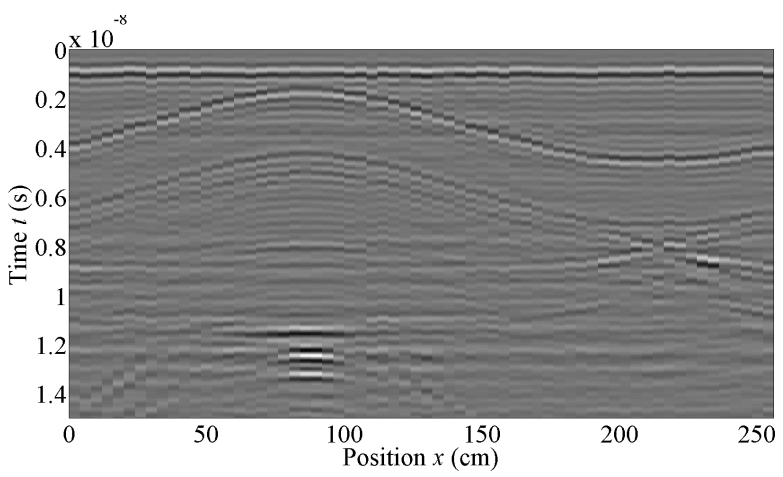

(b)

Fig. 8: B-scans around the tree trunk model. (a) data obtained with the GSSI time-domain radar system with a ground coupled antenna, (b) data obtained with the frequency-domain radar system with an air-coupled TEM horn antenna.

is more uneven due to its progressive static measurement; nevertheless, the reflections seem to be sharper than those in the first GPR image obtained by the commercial radar system. The data acquisition done with a time-domain radar system was, indeed, quicker than the progressive positioning done with the frequency-domain radar system. Nevertheless, it was less comfortable to acquire the data due to the bigger size and weight of the antenna.

\section{REALISTIC REPRESENTATION OF GPR DATA}

In the last experiment, we converted our GPR images from a real tree trunk measurement to a polar representation to express our data in following the geometry of the trunk (see Fig. 9). This kind of GPR representation is very simple to proceed and very practical for a realistic comprehension of the image. Nevertheless, it can be used only for very regular and circular profiles. If this expression is done for an irregular tree trunk cross section, a lot of information of the GPR image will be modified.

To obtain a realistic shape of the tree trunk cross section, photogrammetry using Agisoft Photo Scan was used. This software is based on the structure from motion (SfM) technique which estimates three-dimensional structure from twodimensional pictures. It is necessary to take several photos around the tree trunk (42 in our case), subsequently, the 


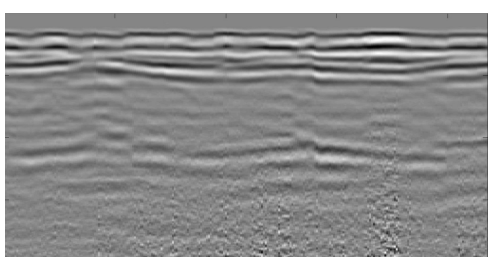

(a)

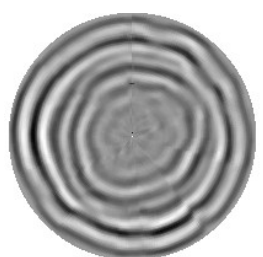

(b)
Fig. 9: (a) B-scan around a real tree trunk, (b) polar representation of the real tree trunk B-scan.

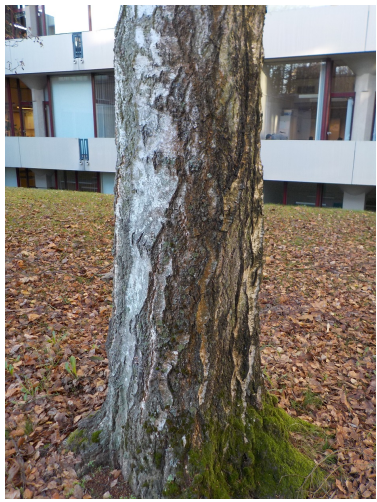

(a)

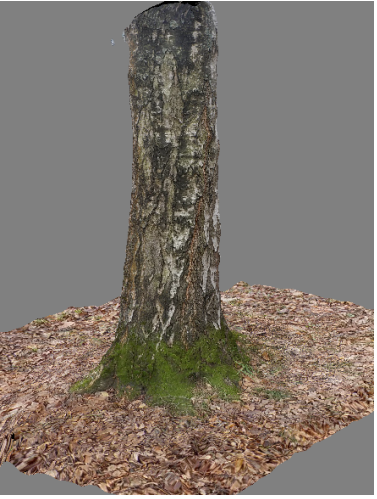

(b)
Fig. 10: (a) photo of a tree, (b) 3-D model of the tree.

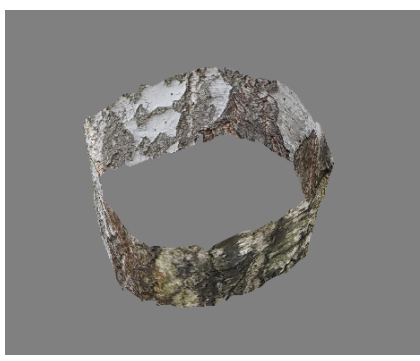

(a)

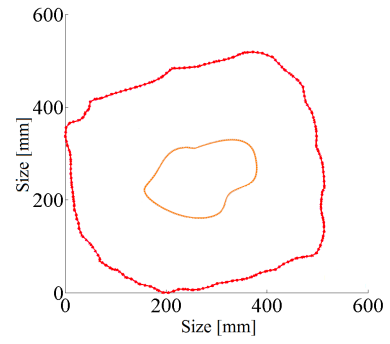

(b)
Fig. 11: (a) 3D section of the tree, (b) contour of the tree trunk cross section and a void inside.

photos must be uploaded to the Agisoft and then, the point cloud of the tree is created. The quality of the 3-D model is proportionally related to the number of uploaded photos. Fig. 10a shows one of our photos of a testing tree (birch) in the campus of the Universitee catholique de Louvain (UCL) and Fig. 10b is a 3-D model of the tree.

The area of interest can be selected from the 3-D model (see Fig. 11a) and the coordinates of its points can be exported for example to the text or the CAD format. In Fig. 11b the real size contour of the real cross section with a simulated void in Matlab is displayed.

Fig. 12a shows a simple simulated radar profile around our testing tree trunk with an artificial internal void. The

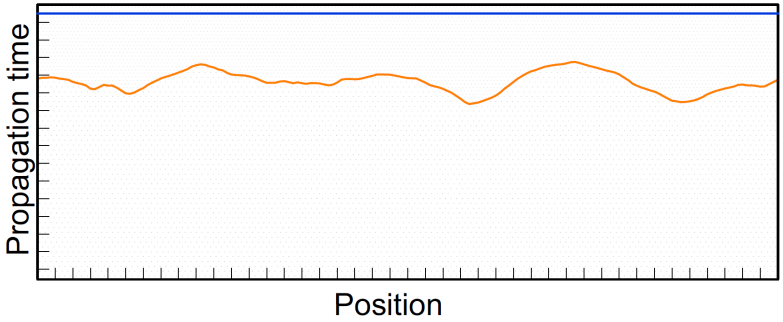

(a)

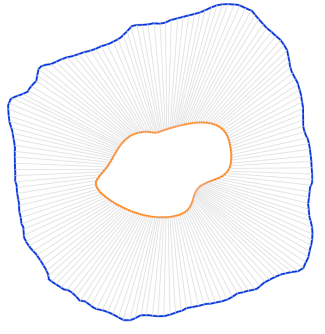

(b)

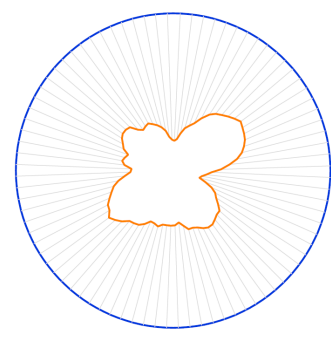

(c)
Fig. 12: (a) B-scan around a real tree, (b) real contour B-scan expression, (c) polar B-scan expression.

blue line is the surface reflection and the orange line is a void reflection. To better understand this GPR image, its real contour and its polar representation was done (Fig. 12b, Fig. 12c). Both images show the internal void, nevertheless, the polar representation strongly changes its real shape.

\section{CONCLusion}

A new custom TEM horn antenna was made in order to make GPR tree trunk testing easier. Its simulated S11 was comparable with the results of the calibration, thus the antenna was built properly according to the design. In order to test the antenna, a radar profile over a sandbox with buried pipes was done. The antenna, combined with a frequency-domain system, satisfactorily detected all of them. Finally, a radar profile around a lab tree model was done in order to compare the measurement with the previous data performed by GSSI time-domain radar system. The biggest advantage of the GSSI system was a fast data acquisition and a solid detection of further objects. However, the new TEM horn antenna was lighter and had a more convenient shape for the tree trunk tomography, thus, the data acquisition was easier. The curves in the GPR image obtained by the TEM horn antenna appear to be sharper.

A realistic contour representation was discussed and analysed and a protocol of its expression was proposed. It was demonstrated that the real contour expression of the GPR image is very important to get the correct shape of the internal void. Other experiments with the non-linear expression of the GPR image will be studied by our team in next months. 


\section{ACKNOWLEDGMENT}

The authors would like to acknowledge the support of the Fonds de la Recherche Scientifique (FNRS), Belgium, through the SENSWOOD project (Convention $\mathrm{n}^{\circ}$ 19526260). This research was also carried out within the framework of EU funded COST Action TU1208 Civil Engineering Applications of Ground Penetrating Radar.

\section{REFERENCES}

[1] J. Ježová and S. Lambot, "Reflection waveforms occurring in gpr tree trunk testing," 2016 16th International Conference on Ground Penetrating Radar (GPR), pp. 1-5, June 2016.

[2] M. Pastorino, A. Randazzo, A. Fedeli, A. Salvadè, S. Poretti, M. Maffongelli, R. Monleone, and M. Lanini, "A microwave tomographic system for wood characterization in the forest products industry," Wood Material Science \& Engineering, vol. 10, no. 1, pp. 75-85, 2015.

[3] J. Ježová, L. Mertens, and S. Lambot, "Ground-penetrating radar for observing tree trunks and other cylindrical objects," Construction and Building Materials, vol. 123, pp. 214-225, 2016.

[4] A. P. Annan, "Gpr - history, trends, and future," Subsurface Sensing Technologies and Applications, vol. 3, no. 4, pp. 253-270, 2002.

[5] M. Solla, H. Lorenzo, F. Rial, and A. Novo, "Ground-penetrating radar for the structural evaluation of masonry bridges: Results and interpretational tools," Construction and Building Materials, vol. 29, pp. $458-465,2012$.

[6] R. D. Bold, G. OConnor, J. Morrissey, and M. Forde, "Benchmarking large scale gpr experiments on railway ballast," Construction and Building Materials, vol. 92, pp. 31 - 42, 2015.

[7] K. Labropoulos and A. Moropoulou, "Ground penetrating radar investigation of the bell tower of the church of the holy sepulchre," Construction and Building Materials, vol. 47, pp. 689 - 700, 2013.

[8] S. Lambot, L. Weihermüller, J. A. Huisman, H. Vereecken, M. Vanclooster, and E. C. Slob, "Analysis of air-launched ground-penetrating radar techniques to measure the soil surface water content," Water Resources Research, vol. 42, no. 11, pp. n/a-n/a, 2006.

[9] J. Minet, P. Bogaert, M. Vanclooster, and S. Lambot, "Validation of ground penetrating radar full-waveform inversion for field scale soil moisture mapping," Journal of Hydrology, vol. 424425, pp. 112 - 123, 2012.

[10] S. A. Al Hagrey, "Geophysical imaging of root-zone, trunk, and moisture heterogeneity," Journal of Experimental Botany, vol. 58, no. 4, pp. 839854, 2007.

[11] J. A. G. Malherbe and N. Barnes, "TEM Horn Antenna with an Elliptic Profile," Microwave and Optical Technology Letters, vol. 49, no. 12, pp. $1548-1551,2007$.

[12] F. Karshenas, A. R. Mallahzadeh, and A. Imani, "Modified TEM horn antenna for wideband applications," Proceedings - 2009 13th International Symposium on Antenna Technology and Applied Electromagnetics and the Canadian Radio Sciences Meeting, ANTEM/URSI 2009, pp. 610, 2009.

[13] B. Scheers, "Development of dielectric-filled tem horn antennas for uwb gpr," Simulation, no. 1, pp. 2-5.

[14] S. Lambot, E. C. Slob, I. D. Van Bosch, B. Stockbroeckx, and M. Vanclooster, "Modeling of ground-penetrating radar for accurate characterization of subsurface electric properties," IEEE Transactions on Geoscience and Remote Sensing, vol. 42, no. 11, pp. 2555-2568, 2004.

[15] A. R. Mallahzadeh and F. Karshenas, "Modified Tem Horn Antenna for Broadband Applications," Progress In Electromagnetics Research, vol. 90 , pp. 105-119, 2009 . 